\title{
Design and Optimization of a Turbine Intake Structure
}

P. Fošumpaur, F. Čihák

The appropriate design of the turbine intake structure of a hydropower plant is based on assumptions about its suitable function, and the design will increase the total efficiency of operation. This paper deals with optimal design of the turbine structure of run-of-river hydropower plants. The study focuses mainly on optimization of the hydropower plant location with respect to the original river banks, and on the optimal design of a separating pier between the weir and the power plant. The optimal design of the turbine intake was determined with the use of 2-D mathematical modelling. A case study is performed for the optimal design of a turbine intake structure on the Nemen river in Belarus.

Keywords: run-of-river hydropower plant, turbine intake, 2-D modelling, optimization.

\section{Introduction}

A suitable design for the water power plant inlet is a basic condition for the proper functioning of the plant, and increases the overall efficiency, optimizing the operation of the plant. The hydraulic solution for the separating pier, and also for other components of the inlet, usually employs mathematical modelling.

The objective of this study is to optimize the hydrodynamic conditions in the area of the inlet of a running water power plant using 2-D mathematical modelling of the flux. The main emphasis is placed on the design of the separating pier between the weir and the power plant, since the wrong width and shape may cause detachment of the current from the face side of the pier, infringing the flux. This disturbance then propagates to the inlet of the nearest aggregate. Measurements conducted on completed water works have demonstrated a decrease in the efficiency of such an aggregate by as much as $30 \%$ [3]. This methodology can also be used for optimization of pier shapes, in order to direct the inlet flux towards the individual turbine blocks. These piers also serve as supports for coarse screens and for the footbridge.

\section{Methods}

The modelling was carried out on the assumption of a stable two-dimensional flux of incompressible fluid. The viscosity and the density were considered equal within the whole area. The basic description of the fluid flux is given by the two Navier-Stokes equations [5]

$$
\frac{\partial u_{i}}{\partial t}+\frac{\partial u_{i}}{\partial x_{j}} u_{j}=-\frac{1}{\rho} \frac{\partial p}{\partial x_{i}}+\frac{\mu}{\rho} \nabla^{2} u_{i}
$$

and the continuity equation

$$
\nabla \mathbf{u}=0
$$

where $u_{i}$ is the velocity vector component in the direction of the $x_{i}$ axis,

$$
\begin{array}{ll}
\rho & \text { density }\left[\mathrm{m}^{3} \cdot \mathrm{s}^{-1}\right], \\
p & \text { hydrostatic pressure }[\mathrm{Pa}], \\
\mu & \text { dynamic viscosity }[\mathrm{Pa} \cdot \mathrm{s}] .
\end{array}
$$

The above set of equations is used for calculating of the laminar flux of a real fluid. For real flows, the current must be considered turbulent. Mathematical modelling of turbulent flow uses the turbulent $k-\varepsilon$ model [6], comprising modified
Navier-Stokes equations with the Reynolds number, the continuity equation, transport equations and the diffusion of kinetic turbulent energy, and equations describing the transport and diffusion of the speed of the dissipation of the kinetic energy of the turbulence.

Equations describing the turbulent flux introduce the time-averaged velocity. The Navier-Stokes equations then take the following form:

$\frac{\partial \overline{\mathbf{u}}}{\partial t}+\overline{\mathbf{u}} \cdot \nabla \overline{\mathbf{u}}=-\frac{1}{\rho} \nabla p+\nabla\left[\left(\frac{\mu}{\rho}+\frac{C_{\mu}}{\sigma_{k}} \frac{k^{2}}{\varepsilon}\right) \cdot \nabla \overline{\mathbf{u}}\right]$.

The equation of turbulent kinetic energy $k$ is

$$
\begin{aligned}
& \rho \frac{\partial k}{\partial t}-\nabla\left[\left(\mu+\rho \frac{C_{\mu}}{\sigma_{k}} \frac{k^{2}}{\varepsilon}\right) \cdot \nabla k\right]+\rho \overline{\mathbf{u}} \cdot \nabla k \\
& =\rho C_{\mu} \frac{k^{2}}{\varepsilon}(\nabla \overline{\mathbf{u}})^{2}-\rho \varepsilon .
\end{aligned}
$$

And the equation of turbulent energy dissipation $\varepsilon$ :

$$
\begin{aligned}
& \rho \frac{\partial \varepsilon}{\partial t}-\nabla\left[\left(\mu+\rho \frac{C_{\mu}}{\sigma_{\varepsilon}} \frac{k^{2}}{\varepsilon}\right) \cdot \nabla \varepsilon\right]+\rho \overline{\mathbf{u}} \cdot \nabla \varepsilon \\
& =\rho C_{\varepsilon 1} C_{\mu} k(\nabla \overline{\mathbf{u}})^{2}-\rho C_{\varepsilon 2} \frac{\varepsilon^{2}}{k} .
\end{aligned}
$$

The $k-\varepsilon$ model contains the following constants, whose values are based on hydraulic model research:

\begin{tabular}{|c|c|c|c|c|}
\hline$C_{\mu}$ & $C_{\varepsilon 1}$ & $C_{\varepsilon 2}$ & $\sigma_{k}$ & $\sigma_{\varepsilon}$ \\
\hline 0.09 & 0.1256 & 1.92 & 1.0 & 1.3 \\
\hline
\end{tabular}

The above-specified equations for 2-D turbulent flow were approximated by the Finite Element Method in the FEMLAB computing environment. The geometric limit conditions are given by the shape of the area of flux, and are based on the layout solution of the inlet to the water power plant. The geometry of the inlet was estimated first, with subsequent optimization of the geometrical solution with the aim of achieving the most uniform possible velocity profile between the individual turbine blocks. The boundary conditions of the flow were selected as follows:

- zero velocity assumed at the walls,

- uniform velocity profile assumed at the inlet boundary,

- zero pressure assumed for the outflow boundary. 


\section{Case study}

The case study of inlet optimization before the turbine blocks is calculated for a power plant project on the Nemen River in Belarus. Fig. 1 shows the layout solution. The flux area was derived from the layout solution of the power plant and the weir. The positioning of the power plant, weir fields and piers between individual turbine blocks was retained. The water power plant comprises a total of five turbine blocks, separated in the inlet by piers with elliptical pier heads. The number of finite elements in the area was 5,000 to 10,000 depending on the alternative and the type of flow. A reliable description of the inlet flow to the power plant required the selection of a sufficiently large area for the solution. The power plant is located on the concave side of a curve with a diameter of approximately $340 \mathrm{~m}$. The area of the solution starts at the beginning of this curve in the vicinity of the upper dock of the lock chamber, where uniform distribution of velocities is assumed.

A total of five basic scenarios of the power plant inlet were examined. Four of them focused on the shape of the separating pier, and one examined the effects of the shape of the side pier between the power plant and the riverbank. The circum- fluence around the separating pier between the power plant and the weir is unsymmetrical; therefore the shape of the pier should be equally unsymmetrical and streamlined. A model study [1,2] demonstrated that the width of the separating pier is a function of turbine flow rates $Q_{T}$. The minimum width of the separating pier is established by the formula:

$$
d=f\left(Q_{T}\right)=C \cdot Q_{T}^{2 / 5}
$$

where factor $C$ depends on the conditions of the pier circumfluence and its values fall within the interval from 0.7 to 1.4 according to study [2]. The specific value of factor $C$ depends on the layout of the water power plant and its positioning in relation to the river flow and the bank, as shown in Fig. 1.

In terms of the design of the separating pier, the following features are fundamental:

$r$ radius of the river course curve above the weir and the power plant,

$b_{1}$ width of the riverbed above the weir and the power plant,

$b_{2}$ width of the power plant,

$l_{2}$ length of the separating pier above the weir,

$\alpha$ central angle of the riverbed, being positive for a power plant on the concave bank and negative if the power plant is located on the convex bank.

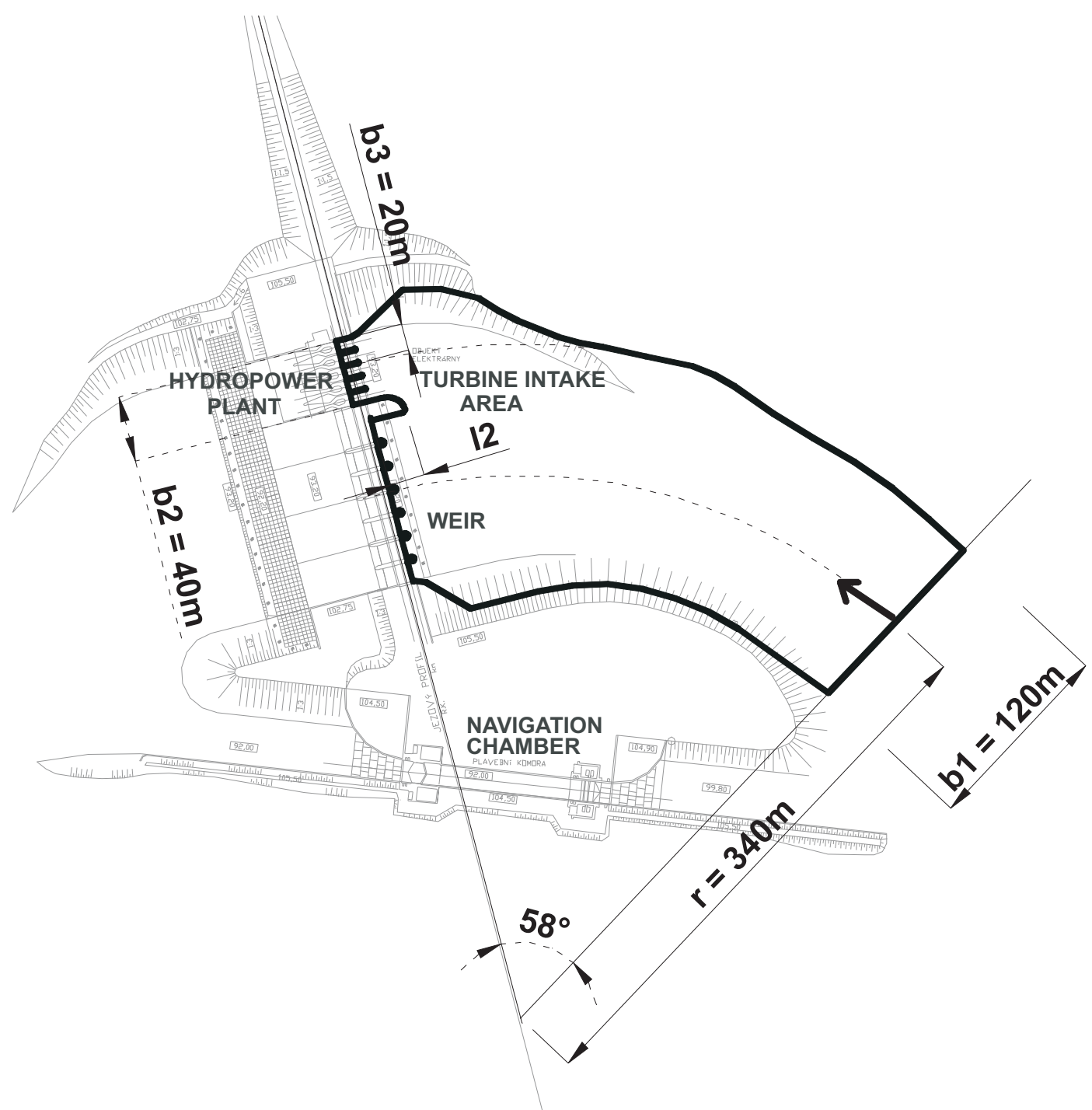

Fig. 1: Layout of the inlet solution of a water power plant on the Nemen River (Belarus) 
Factor $C$ can then be read from the nomogram according to [2], or can be determined from the formula according to Holata [3]:

$$
\begin{aligned}
C= & 0.5\left(1-\frac{l_{2}}{b_{2}} 0.8\right)\left[0.619 \frac{\alpha}{90}\left(\frac{r}{b_{1}} 0.0455-1\right)-1\right] \\
& +0.05 \frac{b_{3}}{b_{2}}+1.5
\end{aligned}
$$

The design of the separating pier of width $d$ employed an elliptical layout according to Fig. 2 [2]. The pier head is composed of an ellipse $(a=0.834 d ; b=0.166 d)$ and two circular curves of radius $0.24 d$, and $0.12 d$ respectively. The angle $\phi$ of the central circle curve is:

$$
\phi=\arcsin \left(\frac{2 \times 0.166 d}{0.24 d}-1\right)=22.54^{\circ}
$$

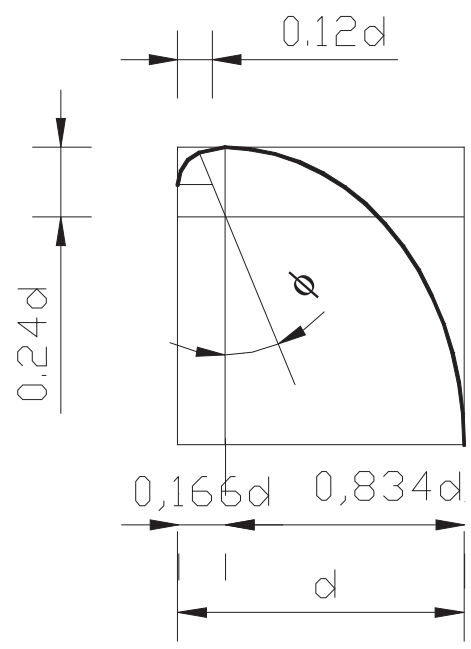

Fig. 2: Elliptical shape of the separating pier

Scenario 1 This scenario is based on the length of the separating pier above the weir $l_{2} \cong b_{2} / 2$. The bank-side pier creates an angle of $45^{\circ}$ with the axis of the river flow within the weir profile.

Scenario 2 Since calculations showed that the angle of the bank-side pier of $45^{\circ}$ negatively affects the field of flow above the aggregate near the riverbank, this scenario used an angle of $30^{\circ}$. The layout of all the other elements remains unchanged from scenario 1 . All subsequent scenarios have the bank-side pier angle $30^{\circ}$. A chart of scenario 2 is given in Fig. 3.

Scenario 3 This scenario uses the length of the face side of the pier above the weir $l_{2} \cong b_{2} / 4$.

Scenario 4 This alterative represents the greatest protrusion of the separating pier above the weir examined here. The protrusion is based on the maximum allowable width of the separating pier, determined according to the width of the weir field, as stated in the input data $(12 \mathrm{~m})$. This is the upper limit of the pier protrusion above the weir.
Scenario 5 The last investigated scenario is based on scenario 3. The width of the separating pier in both cases is $10.2 \mathrm{~m}$. The scenarios differ in the shape of the pier head area, which in this scenario is based on a logarithmic spiral layout.

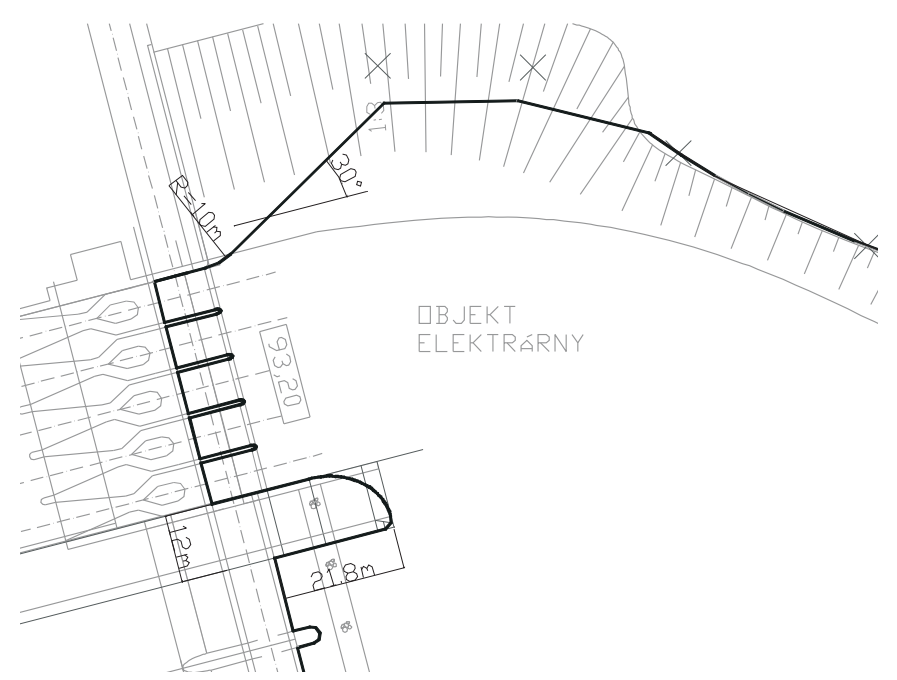

Fig. 3: Scenario 2

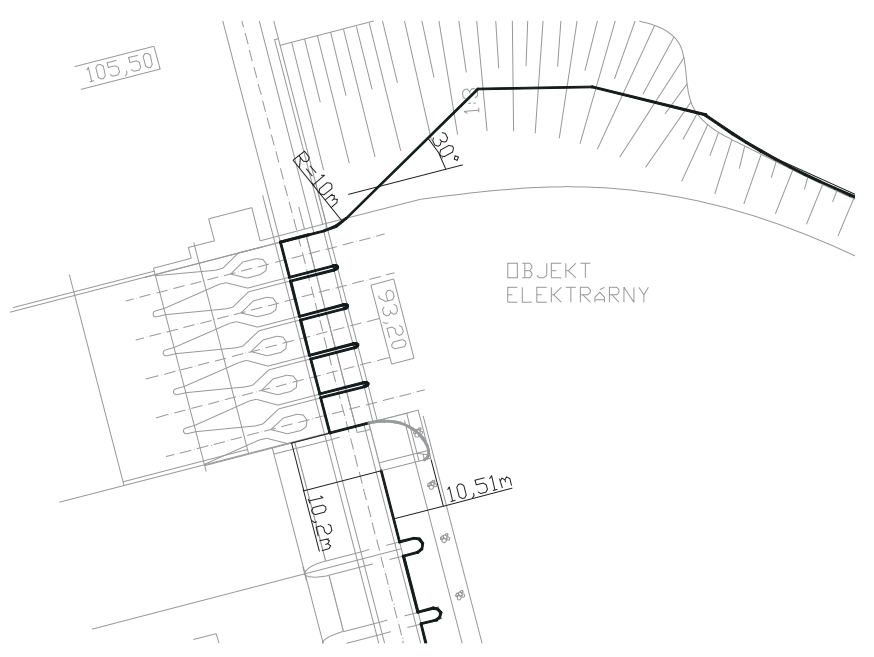

Fig. 4: Scenario 3

The logarithmic spiral function for shaping the separating pier for scenario 5 was used in the following form:

$$
\rho=2.344 d \log \frac{180}{\phi}
$$

where $\rho$ is the polar vector $[\mathrm{m}], \phi$ denotes the polar angle $\left[^{\circ}\right]$ and $d$ is the pier width [m]. Fig. 6 shows a chart of the pier layout in comparison to scenario 3. The drawing also indicates the beginning of the logarithmic spiral.

Fig. 7 and Fig. 8 show isosurfaces for total velocities and streamlines for scenarios 1 and 5 under laminar flow for Reynolds number $\operatorname{Re}=2000$. It holds that the streamlines follow the optimum (streamlined) path under potential laminar flow. Any differences between this ideal shape determined 


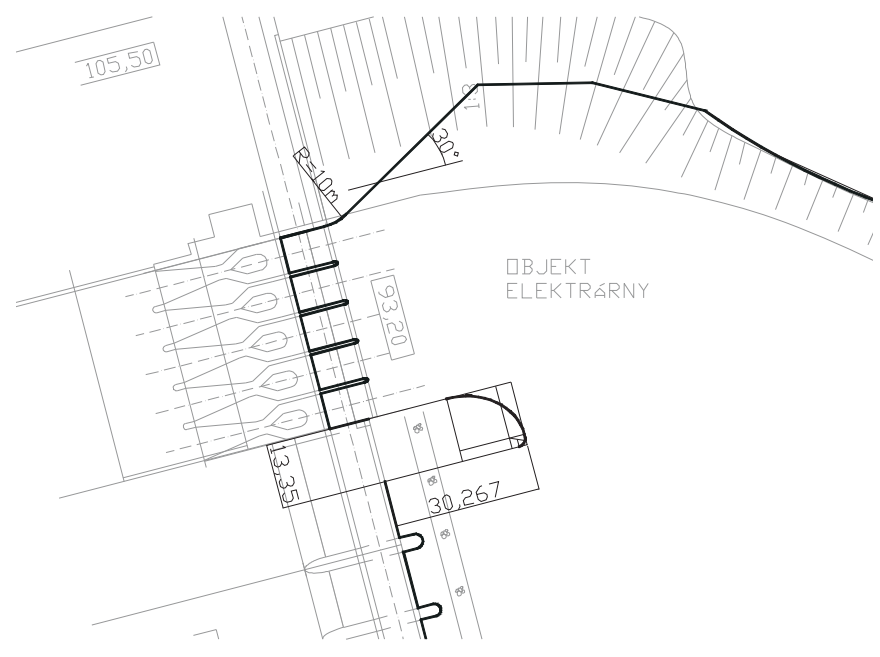

Fig. 5: Scenario 4

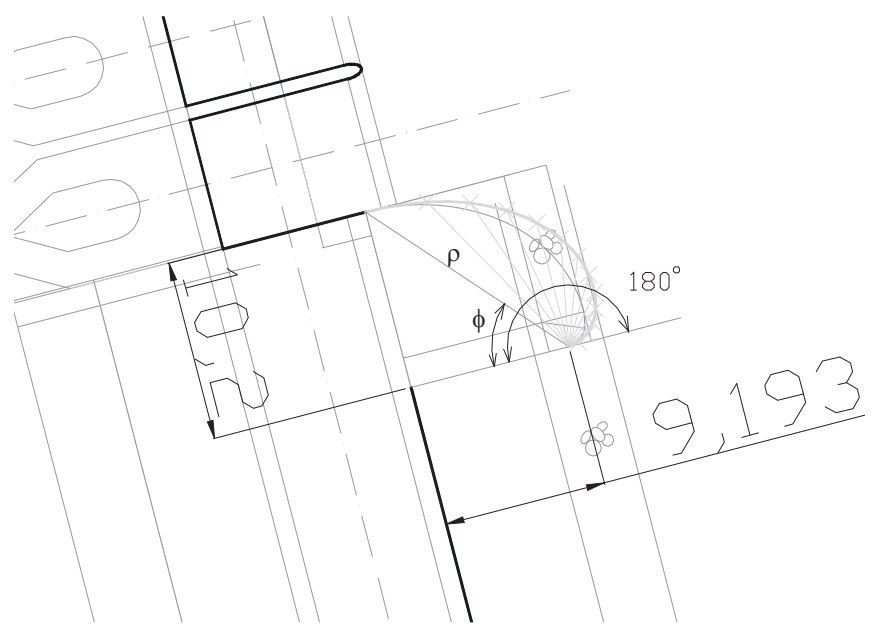

Fig. 6: Scenario 5

by the streamlines and the actual shape of the structures subject to circumfluence thus indicate shortcomings in the shape design. This analysis was performed for the whole investigated area; however, for the sake of greater clarity only a detail of the separating pier is shown.

The analysis made it clear that the most suitable solutions are offered by scenarios 3 and 5 (the least protrusion of the pier above the weir), where the streamlines and the contour of the separating pier are virtually parallel, making it safe to assume no detachment of the flow from the face side of the

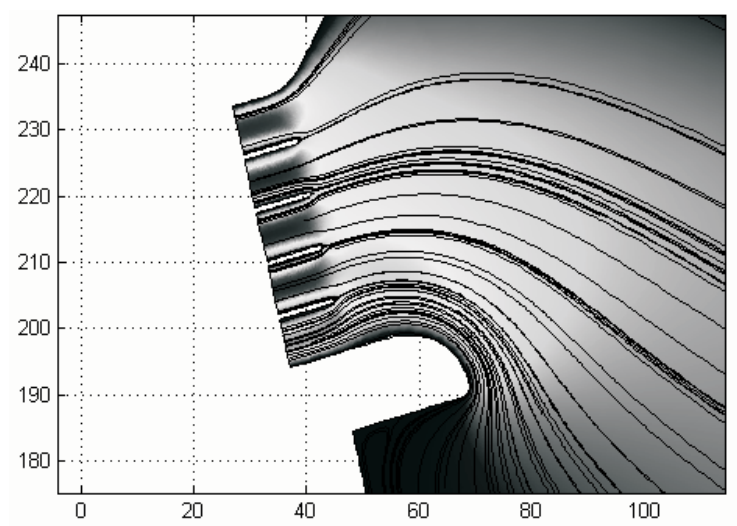

Fig. 7: Scenario 1: laminar flow

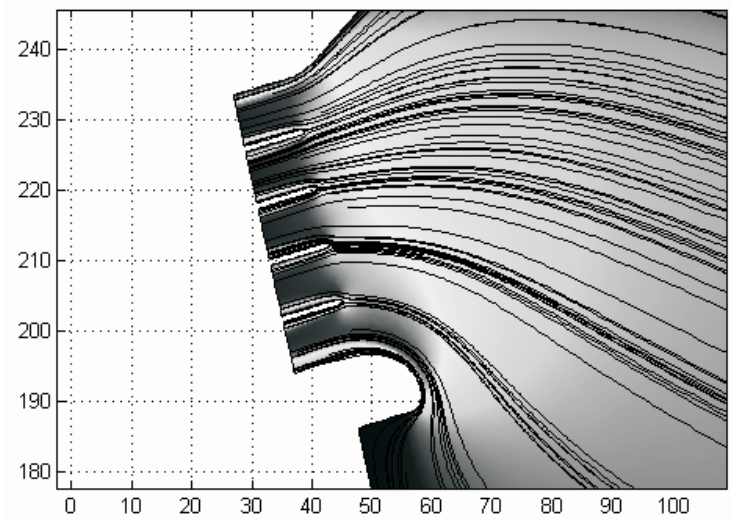

Fig. 8: Scenario 5: laminar flow

structure. Conversely, the worst results were obtained for the pier shapes in scenarios 1 and 2 (the scenarios differ in the shape of the side pier only). Nor is the pier according to scenario 4 (the greatest length protruding above the weir) ideal in terms of the streamlines.

The following analysis compares the individual scenario solutions for actual flow rate values under turbulent flux. The results of this investigation enable the direct selection of the optimum scenario based on the characteristics of the velocity field at the inlet of the individual turbine blocks. Figs. 9 and 10 show a comparison between the velocity fields for scenarios 3 and 4 . The charts clearly demonstrate that in the case of scenario 3 the flux field above the turbines is more uniform than in the case of scenario 4 , where the velocities in the vicin-

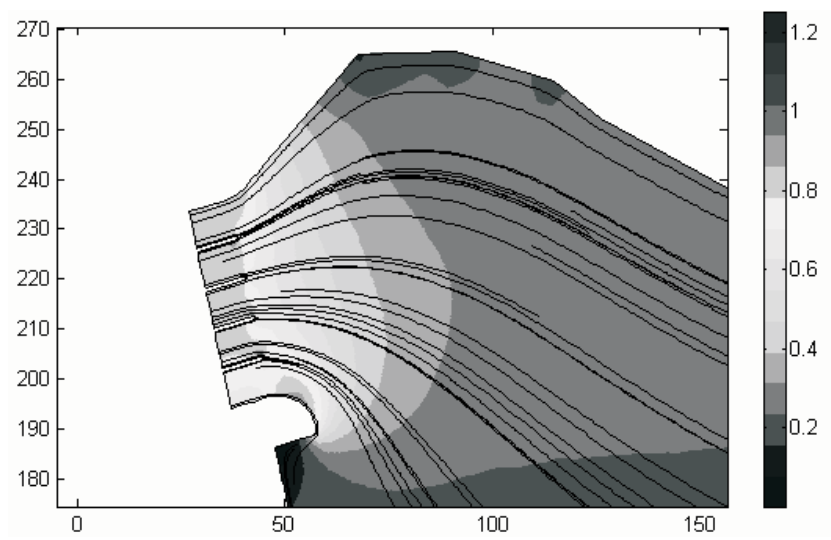

Fig. 9: Scenario 3: total velocity $\left[\mathrm{m} \cdot \mathrm{s}^{-1}\right]$ and streamlines

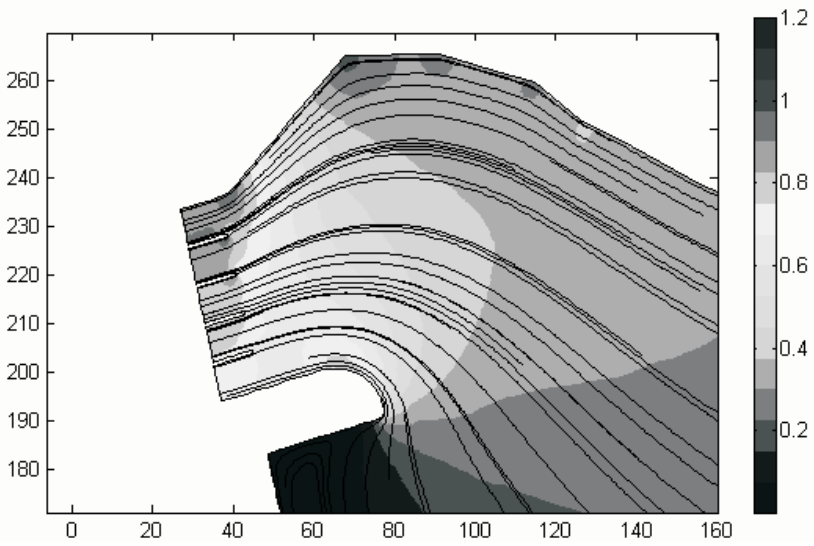

Fig. 10: Scenario 4: total velocity $\left[\mathrm{m} \cdot \mathrm{s}^{-1}\right]$ and streamlines 


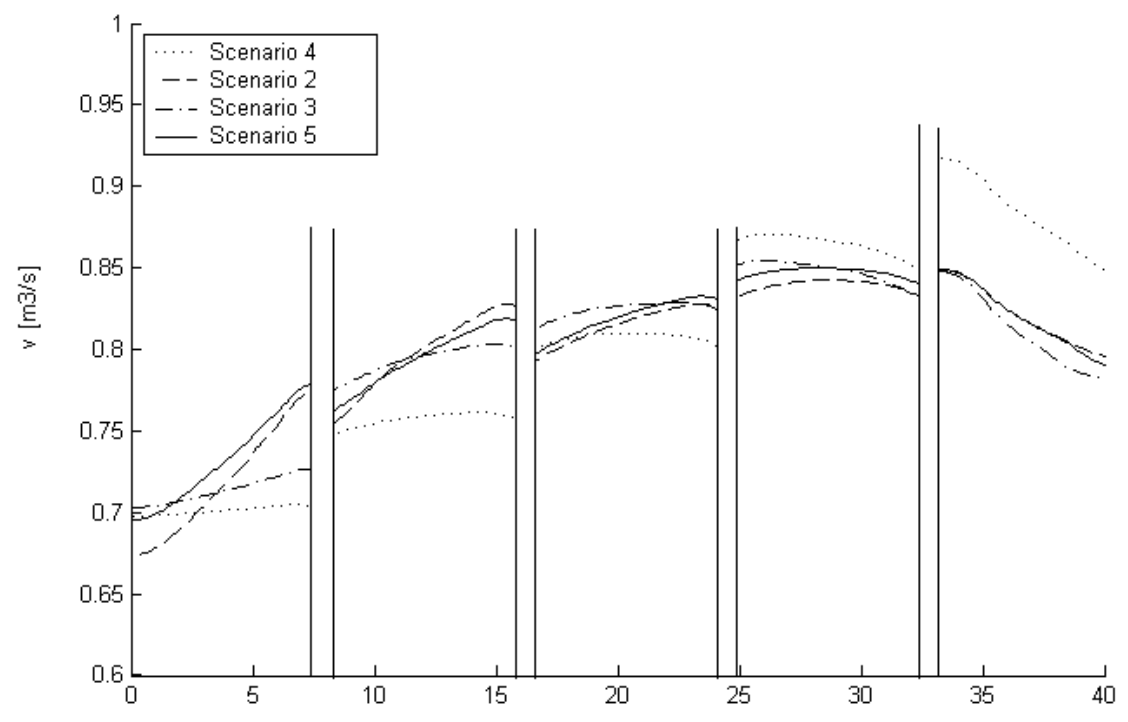

Fig. 11: Distribution of velocities above the turbines for individual scenarios

ity of the bank-side pier are significantly higher than behind the separating pier between the power plant and the weir.

A comparison of the scenarios of the inlet solution is shown in Fig. 11. The graph shows the velocity profile in a cross section above the turbines (on the outflow limit of the investigated area). Scenarios with differing shapes of the separating pier are monitored. Scenario 1 with side pier angle $45^{\circ}$ was replaced by the more suitable scenario 2 , with pier angle $30^{\circ}$. Based on the analysis, scenario 3 was recommended as the design solution for the shape of the separating pier.

\section{Conclusion}

There are numerous empirical relations and recommendations for the optimum design of hydrodynamic shapes of elements subject to circumfluence in the area of the inlets of water power plants, usually based on physical research in laboratories. This paper utilises these recommendations in the formulation of the initial design scenarios, which are subsequently optimised using 2-D mathematical modelling of the laminar and turbulent flux. The advantage of this solution is above all in the low time and financial demands of the optimization of inlet elements of water power plants.

\section{Acknowledgments}

This research has been supported by grant No. 103/02/D049 of the Grant Agency of the Czech Republic.

\section{References}

[1] Čihák, F., Medřický, V.: Weir Design. Praha: Vydavatelství ČVUT, 1991.

[2] Gabriel, P., Grandtner, T., Průcha, M., Výbora, P.: Weirs. Praha: SNTL, 1989.

[3] Holata, M.: Small Hydro-Power Plants. Ed.: Pavel Gabriel, Praha: Academia, 2002.

[4] Hunter, S. C.: Mechanics of Continuous Media. Chichester (England): Ellis Horwood, 1976.

[5] Kolář, V., Patočka, C., Bém, J.: Hydraulics. Praha: SNTL, 1983.

[6] Wilcox, D.: Turbulence Modeling for CFD. $2^{\text {nd }}$ edition, DCW Industries Inc., 1998.

Dr. Ing. Pavel Fošumpaur

phone: +420224354425

e-mail: fosump@fsv.cvut.cz

Prof. Ing. František Čihák, DrSc.

phone: +420224354611

e-mail: cihak@fsv.cvut.cz

Department of Hydrotechnics

Czech Technical University of Prague

Faculty of Civil Engineering

Thákurova 7

16629 Prague 6, Czech Republic 